\title{
Assessing the Mental Health Literacy of Secondary School Educators
}

\author{
Marco R Publico \\ Philippine Military Academy
}

\begin{abstract}
Mental health remains to be one of the most pressing concerns across the world, and teachers play an influential role in the lives and learning of students struggling with mental health. Unfortunately, teachers have expressed concerns of feeling overwhelmed and unprepared to handle the mental health needs of their students because of the lack of knowledge, skills, competence, and resources. This research was conducted to provide a systematic appraisal of the mental health literacy among secondary school teachers. Utilizing the Education-modified Mental Health Literacy Scale which was adapted by Kenney from the work of O'Connor, eighty-one (81) teachers from public and private secondary schools were surveyed in six domains, to include: disorder recognition scale, risk factor knowledge scale, self-treatment knowledge scale, available professional help scale, information seeking knowledge scale, and attitudes scale. It was found that overall, the participants manifested a high level of mental health literacy, and are most adept at the attitudes scale. Conversely, it was found that teachers need more knowledge and training in the risk-factor knowledge scale. No statistically significant difference was found in the mental health scale scores when compared between schools and grade level. The researcher recommends using the results of the current study as a springboard for crafting a professional development program that focuses on increasing the literacy of teachers about mental health.
\end{abstract}

Keywords: Mental Health Literacy, School-Based, Teachers, Professional Development Program

info@icate.org 